\title{
Bistability of ferroelectric domain walls: Morphotropic boundary and strain effects
}

\author{
P. V. Yudin,* A. K. Tagantsev, and N. Setter \\ Ceramics Laboratory, Swiss Federal Institute of Technology (EPFL), CH-1015 Lausanne, Switzerland
}

(Received 10 May 2013; published 8 July 2013)

\begin{abstract}
The internal structure of neutral $180^{\circ}$ domain walls in perovskite-type ferroelectrics is studied in terms of Landau theory taking into account electromechanical coupling. The study is focused on the wall bistability, a factor of potential interest for information storage. A strong impact of elastic effects on the wall structure is demonstrated. It is shown that the conclusion derived earlier by Houchmandzadeh et al. [J. Phys.: Condens. Matter 3, 5163 (1991)], neglecting the electrostictive coupling, that all the domain walls near the boundary between two ordered phases become bistable may not hold due to the elastic effects. Criteria for domain-wall bistability are formulated in terms of the materials thermodynamic properties and the wall orientation. The obtained general results are applied to the analysis of bistability of $180^{\circ}$ domain walls in $\mathrm{Pb}\left(\mathrm{Zr}_{c}, \mathrm{Ti}_{1-c}\right) \mathrm{O}_{3}$ near the tetragonal-rhombohedral morphotropic boundary. It is shown that, on the tetragonal side, the electrostrictive interaction suppresses the wall bistability that was predicted in terms of the theory neglecting the elastic effects. On the rhombohedral side, the domain walls are found bistable or not depending on the anisotropy of the correlation energy, the information on which is not presently available. It is also shown that, in the rhombohedral phase, the anisotropy of the correlation energy results in appearance of additional polarization component in the plane of the wall.
\end{abstract}

DOI: 10.1103/PhysRevB.88.024102

PACS number(s): 77.80.Dj, 77.80.bn, 99.10.Fg

\section{INTRODUCTION}

Domain walls in ferroelectrics are of high interest to both fundamental and applied research. This interest is first explained by their paramount role for the switching processes in multidomain materials, as well as by a considerable contribution that they make to dielectric, piezoelectric, transport, and other macroscopic properties of ferroelectrics. ${ }^{1}$ With advances in nanotechnology, domain walls (DWs) start being considered as individual objects rather than just boundaries separating two domains. It has been shown that certain domain boundaries offer unique properties that are not exhibited in the bulk of the ferroic sample. ${ }^{2-4}$ The intention to create nanodevices on the basis of domain walls motivates further fundamental research of their internal structures. Of special interest are theoretical results predicting bistable domain walls ${ }^{5,6}$ where information may be potentially stored. This type of wall may exist between two oppositely poled domains $\left(180^{\circ} \mathrm{DW}\right)$. The bistability occurs when the domain-wall profile loses inversion symmetry with respect to the wall center (centrosymmetry). The basic solution for polarization in the $180^{\circ} \mathrm{DWs}$ is the so-called Isingprofile, in which a single polarization component $P_{1}$ is present [Fig. 1(a)]; this solution is centrosymmetric. However, at some material parameters an additional polarization component $P_{2}$ may arise, breaking the centrosymmetry of the wall. ${ }^{6}$ The resulting two-component solution for the polarization is referred to as Bloch wall; its profile is shown in Figs. 1(b) and 1(c). The Bloch wall has two energetically equivalent states, corresponding to the left-handed and right-handed polarization spirals. Theoretically, the Bloch wall may be switched from one state to the other by application of electric field along the $y$ axis. This feature makes Bloch walls interesting objects from the point of view of dense memory applications. To the best of our knowledge the only material where Bloch walls are predicted is the rhombohedral phase of $\mathrm{BaTiO}_{3},{ }^{5-8}$ and this is done by numerical simulations, based on LandauGinsburg-Devonshire (LGD) theory and $a b$ initio calculations. However, such domain walls may have limited applications because of difficulties, associated with cryogenic temperatures needed to obtain this phase in the material. Thus, of interest is to investigate the possibility of bistable $180^{\circ}$ walls in other ferroelectrics, in particular at room temperature.

Bloch walls appear when Ising solution becomes unstable with respect to appearance of a nonzero $P_{2}$ component. The question of stability of Ising walls was earlier studied theoretically using a simple model neglecting electromechanical coupling. ${ }^{9,10}$ In that framework, a conclusion may be drawn that at the vicinity of the boundary between two different ordered phases, any $180^{\circ}$ domain wall becomes of Bloch type. ${ }^{10}$ This would suggest, for example, bistability of $180^{\circ}$ domain walls near tetragonal-rhombohedral morphotropic boundary, making morphographic-boundary solid solutions undisputable candidates for the existence of bistable domain walls. Meanwhile, it is known that electromechanical coupling may radically affect the DW properties. ${ }^{3,11}$ This justifies revisiting the problem of wall bistability incorporating elastic effects in general and in particular in morphotropic-boundary systems.

In this work we explore the stability of Ising walls taking into account electromechanical coupling for the case of a ferroelectric with a cubic centrosymmetric parent phase and tetragonal and/or rhombohedral ferroelectric phases, using a LGD phenomenological model. We consider electrically neutral $180^{\circ}$ domain walls, including walls of low-symmetry orientations. This issue was partially addressed, for the case of the tetragonal crystalline symmetry, in our earlier publication. ${ }^{12}$ Here we consider both tetragonal and rhombohedral phases of the material and put emphasis on the conditions for the occurrence of bistable walls. The paper is organized as follows. A general statement of the problem and governing equations are given in Sec. II. Sections III and IV are devoted to the tetragonal and the rhombohedral phases respectively. In these sections we mainly discuss the stability of Ising walls and formulate conditions under which the Ising wall undergoes a phase transition into the Bloch bistable state. Sections III A and IV A are devoted to 

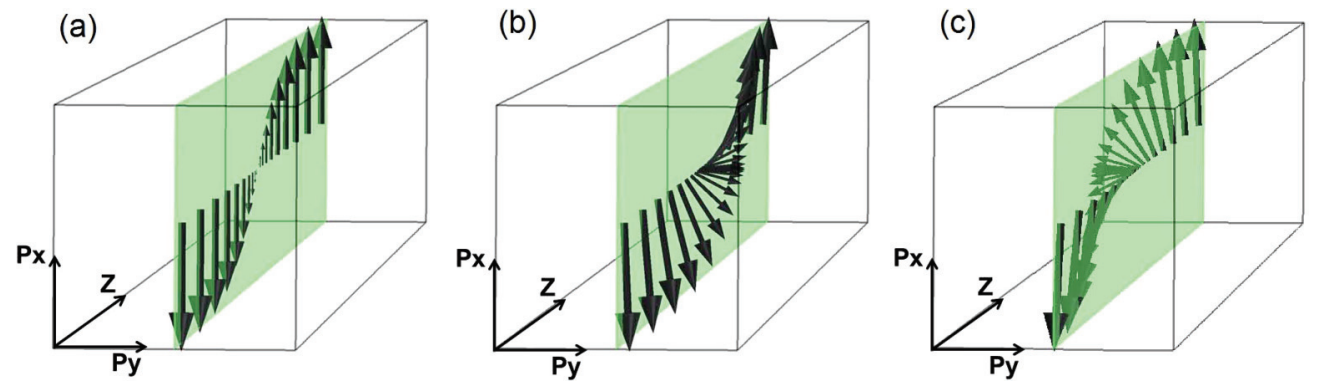

FIG. 1. (Color online) The polarization vector distribution along the normal to the domain wall z. (a) Ising wall. (b), (c) Bistable Bloch wall in the left-handed state (b) and in the right-handed one (c).

the approximation neglecting the electromechanical coupling whereas in Secs. III B and IV B elastic effects are taken into account. Conditions for the wall bistability are formulated in terms of the thermodynamic parameters of the ferroelectric material and the wall orientation. In Secs. III C and IV C the obtained general results are applied to the analysis of bistability of $180^{\circ}$ domain walls in $\mathrm{Pb}\left(\mathrm{Zr}_{c}, \mathrm{Ti}_{1-c}\right) \mathrm{O}_{3}$ (PZT) on the tetragonal and rhombohedral sides of the morphotropic boundary, respectively. In Sec. IV D we consider the question of bistability in domain walls with a more complex structure than that of the Ising wall, which arises for the low-symmetry wall orientations in the rhombohedral materials. A definition of the symbols used in our theory is in Table I.

\section{GIBBS POTENTIAL AND GOVERNING EQUATIONS}

We base our calculations on the Gibbs thermodynamic potential expanded up to the fourth power in polarization, which is sufficient for the description of second-order phase transition ferroelectrics far from the tricritical point,

$$
\begin{aligned}
G= & \frac{1}{2} A_{i j} P_{i} P_{j}+\frac{1}{4} B_{i j k l} P_{i} P_{j} P_{k} P_{l}+\frac{1}{2} D_{i j k l} \frac{\partial P_{i}}{\partial x_{j}} \frac{\partial P_{k}}{\partial x_{l}} \\
& -Q_{i j k l} \sigma_{i j} P_{k} P_{l}-\frac{1}{2} s_{i j k l} \sigma_{i j} \sigma_{k l},
\end{aligned}
$$

where $A_{i j}=\alpha \delta_{i j}$ and $B_{i j k l}=\frac{\beta_{2}}{3}\left(\delta_{i j} \delta_{k l}+\delta_{i k} \delta_{j l}+\delta_{i l} \delta_{j k}\right)+$ $\left(\beta_{1}-\beta_{2}\right) g_{i j k l}$ are the coefficients of the Landau expansion with $\alpha=\alpha_{0}\left(T-T_{c}\right.$ ) ( $T$ and $T_{c}$ are the temperature and the Curie temperature, respectively) and $\beta_{1}>0$ and $\beta_{2}>-\beta_{1} / 2$ to provide the stability of the system. The following notations are also used: $\sigma_{i j}$ is the stress tensor, $\delta_{i j}$ is the invariant Kronecker tensor, and $g_{i j k l}$ is the fourth rank tensor, having the following structure in the cubic crystallographic axes: if $i=$ $j=k=l$ then $g_{i j k l}=1$ and otherwise $g_{i j k l}=0$. Hereafter the summation over repeating indices is implied. The tensors controlling the gradient energy $D_{i j k l}$, the electrostriction $Q_{i j k l}$, and elastic compliance $s_{i j k l}$ are introduced as follows:

$$
\begin{aligned}
D_{i j k l}= & D_{12} \delta_{i j} \delta_{k l}+D_{44}\left(\delta_{i k} \delta_{j l}+\delta_{i l} \delta_{j k}\right) \\
& +\left(D_{11}-D_{12}-2 D_{44}\right) g_{i j k l}, \\
Q_{i j k l}= & Q_{12} \delta_{i j} \delta_{k l}+Q_{44}\left(\delta_{i k} \delta_{j l}+\delta_{i l} \delta_{j k}\right) \\
& +\left(Q_{11}-Q_{12}-\frac{Q_{44}}{2}\right) g_{i j k l},
\end{aligned}
$$

\begin{tabular}{|c|c|}
\hline Symbol & Definition \\
\hline$A_{i, j}$ & Dielectric stiffness tensor \\
\hline$B_{i, j, k, l}$ & 4th-order dielectric stiffness \\
\hline$D_{i j k l}$ & Correlation energy tensor \\
\hline$e_{i j k}$ & Levi-Civita tensor \\
\hline$G$ & Gibbs potential \\
\hline$g_{i j k l}$ & Fourth rank invariant cubic tensor \\
\hline$P_{i}$ & Polarization vector (ferroelectric part) \\
\hline$P_{0 T}$ & $\begin{array}{l}\text { Spontaneous polarization vector in the tetragonal } \\
\text { phase }\end{array}$ \\
\hline$P_{0 R}$ & $\begin{array}{l}\text { Spontaneous polarization vector in the tetragonal } \\
\text { phase }\end{array}$ \\
\hline$Q_{i j k l}$ & Electrostriction tensor \\
\hline$R_{0}$ & $\begin{array}{l}\text { Renormalization coefficient for } 4 \text { th-order } \\
\text { dielectric stiffness }\end{array}$ \\
\hline$s_{i j k l}$ & Compliance tensor \\
\hline$T$ & Temperature \\
\hline$T_{C}$ & Curie temperature \\
\hline$t$ & Domain-wall thickness \\
\hline$U_{i}$ & Displacement vector \\
\hline$x_{i}$ & Cartesian coordinates \\
\hline$x_{C, i}$ & $\begin{array}{l}\text { Cartesian coordinates in crystallographic } \\
\text { reference frame }\end{array}$ \\
\hline$\alpha$ & Inverse dielectric susceptibility \\
\hline$\beta$ & Fourth-order dielectric stiffness coefficients \\
\hline$\Delta$ & Correlation energy tensor anisotropy parameter \\
\hline$\delta_{i j}$ & Invariant Kronecker tensor \\
\hline$\varepsilon_{i j}$ & Strain tensor \\
\hline$\Theta$ & $\begin{array}{l}\text { Renormalization coefficient for 4th-order } \\
\text { dielectric stiffness }\end{array}$ \\
\hline$\lambda_{m}$ & Smallest eigenvalue \\
\hline$\Xi$ & $\begin{array}{l}\text { Renormalization coefficient for 4th-order } \\
\text { dielectric stiffness }\end{array}$ \\
\hline$\sigma_{i j}$ & Mechanical stress \\
\hline$\varphi$ & Tilt angle of the domain wall \\
\hline$\Omega$ & $\begin{array}{l}\text { Renormalization coefficient for 4th-order } \\
\text { dielectric stiffness }\end{array}$ \\
\hline
\end{tabular}

$$
\begin{gathered}
s_{i j k l}=s_{12} \delta_{i j} \delta_{k l}+s_{44}\left(\delta_{i k} \delta_{j l}+\delta_{i l} \delta_{j k}\right) \\
+\left(s_{11}-s_{12}-\frac{s_{44}}{2}\right) g_{i j k l} .
\end{gathered}
$$

Here we use the Voight notations according to the reference text $^{13}$ except for $Q_{44}=4 Q_{2323}$.

TABLE I. Definition of symbols used in this paper. 
From the Gibbs potential (1) one obtains the equations of state

$$
\frac{\partial G}{\partial P_{i}}-\frac{d}{d x}\left(\frac{\partial G}{\partial P_{i}^{\prime}}\right)=0 .
$$

In addition we imply the condition of mechanical equilibrium

$$
\frac{\partial \sigma_{i j}}{\partial x_{j}}=0(i, j=1,2,3),
$$

where $P_{i}$ is the ferroelectric part of the polarization vector (hereafter we use the term polarization as shorthand).

A spatially homogeneous solution to the system of equations (5) and (6) at zero stress yields the parent phase with $P=0$ at $\alpha>0$, the tetragonal phase with the spontaneous polarization $P_{0 T}=\sqrt{\frac{-\alpha}{\beta_{1}}}$, and six equivalent domain states $\left\{P_{0 T}, 0,0\right\},\left\{-P_{0 T}, 0,0\right\},\left\{0, P_{0 T}, 0\right\}$, etc., at $\alpha<0$ and $\beta_{2}>$ $\beta_{1}$, and the rhombohedral phase with spontaneous polarization $P_{0 R}=\sqrt{\frac{-3 \alpha}{\beta_{1}+2 \beta_{2}}}$ and eight equivalent domain states $\left\{\frac{P_{0 R}}{\sqrt{3}}, \frac{P_{0 R}}{\sqrt{3}}, \frac{P_{0 R}}{\sqrt{3}}\right\}, \quad\left\{\frac{P_{0 R}}{\sqrt{3}}, \frac{P_{0 R}}{\sqrt{3}},-\frac{P_{0 R}}{\sqrt{3}}\right\} \ldots\left\{-\frac{P_{0 R}}{\sqrt{3}},-\frac{P_{0 R}}{\sqrt{3}},-\frac{P_{0 R}}{\sqrt{3}}\right\}$, at $\alpha<0$ and $\beta_{2}<\beta_{1}$. The transition between the tetragonal and the rhombohedral phases occurs at the morphotropic boundary, determined by $\beta_{2}=\beta_{1}$.

We consider a one-dimensional problem, describing planar $180^{\circ}$ domain walls with polarization vector $\vec{P}$ and mechanical stresses $\sigma_{i j}$ being dependent only on the coordinate $z$ normal to the plane of the wall. Correspondingly, the boundary conditions are the following:

$$
\vec{P}= \pm\left.\vec{P}_{0}\right|_{z= \pm \infty} ; \quad \sigma_{i j}=\left.0\right|_{z= \pm \infty} \text {. }
$$

Here $\vec{P}_{0}$ is the vector of spontaneous polarization in the domain at $z \rightarrow \infty$. Only electrically neutral domain walls are considered, and we apply the approximation of the absence of the bound charge: $\operatorname{div} \vec{P}=0$. In this model we neglect the flexoelectric coupling. It was recently shown that this coupling may have a nontrivial impact on the domain-wall structure. ${ }^{14,15}$ However, quantitatively, this impact was shown to be relatively small. ${ }^{14}$ In view of this, we omit the flexoelectric coupling in our phenomenological model to have a more transparent picture of the simple and strong elastic effects addressed in this paper.

\section{TETRAGONAL PHASE}

Electrically neutral domain walls are parallel to the spontaneous polarization vector in the domains. For the tetragonal phase considered in this section, possible orientations of such walls are shown in Fig. 2. Here the crystallographic cubic reference is denoted as $\left\{x_{C 1}, x_{C 2}, x_{C 3}\right\}$ while the $\{x, y, z\}$ frame is associated with the wall, where the $x$ axis is directed along the vector of spontaneous polarization in the domains and the $z$ axis is normal to the wall. Hereafter we use the notations $P_{1}$ and $P_{2}$ for the $x$ - and $y$-polarization components, respectively. The third polarization component is zero, as a consequence of Eqs. (7) and $\operatorname{div} \mathbf{P}=0$, which $\operatorname{reads} \frac{d P_{z}}{d z}=0$ in the one-dimensional (1D) case.

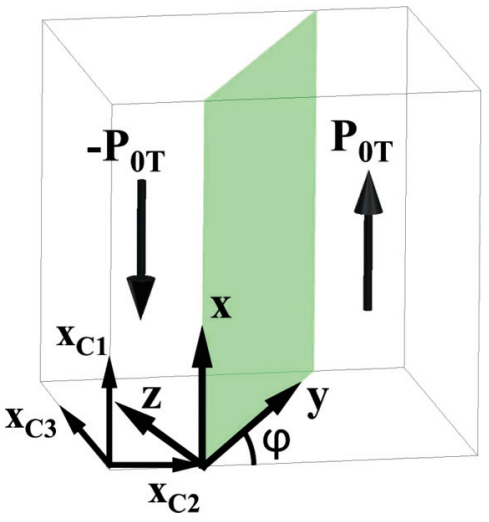

FIG. 2. (Color online) Neutral wall orientations in the tetragonal phase and the corresponding reference frames; $\varphi$ is the dihedral angle between the wall plane and the (001) plane.

\section{A. Approximation neglecting electromechanical coupling}

As a benchmark we consider the problem without taking into account elastic effects, i.e., with $Q_{i j k l}$ and $s_{i j k l}$ tensors set to zero in (1). With these simplifications, the Gibbs potential (1) in the reference frame related to the domain wall takes the form

$$
\begin{aligned}
G_{T}= & \frac{1}{2} \alpha\left(P_{1}^{2}+P_{2}^{2}\right)+\frac{1}{4} \beta_{1} P_{1}^{4}+\frac{1}{2} \beta_{2} P_{1}^{2} P_{2}^{2} \\
& +\frac{1}{4} \beta_{T}\left(P_{2}^{4}\right)+\frac{1}{2} \delta_{1}\left(\frac{d P_{1}}{d z}\right)^{2}+\frac{1}{2} \delta_{2}\left(\frac{d P_{2}}{d z}\right)^{2},
\end{aligned}
$$

where $\beta_{T}=\left[3 \beta_{1}+\beta_{2}+\left(\beta_{1}-\beta_{2}\right) \cos 4 \varphi\right] / 4$,

$$
\begin{gathered}
\delta_{1}=D_{44}, \delta_{2}=\delta_{1}\left(\frac{1+\cos 4 \varphi}{2}+\frac{1-\cos 4 \varphi}{2 \Delta}\right), \\
\Delta=\frac{2 D_{44}}{D_{11}-D_{12}}
\end{gathered}
$$

is the gradient term anisotropy parameter. One readily checks that the tetragonal phase considered in this section corresponds to $\beta_{2} / \beta_{1}>1$.

Using (8), one obtains the following equations of state:

$$
\begin{aligned}
& \alpha P_{1}+\beta_{1} P_{1}^{3}+\beta_{2} P_{1} P_{2}^{2}-\delta_{1} \frac{\partial^{2} P_{1}}{\partial z^{2}}=0, \\
& \alpha P_{2}+\beta_{T} P_{2}^{3}+\beta_{2} P_{2} P_{1}^{2}-\delta_{2} \frac{\partial^{2} P_{2}}{\partial z^{2}}=0 .
\end{aligned}
$$

The Ising wall is a solution to Eqs. (11) with the only one nonzero polarization component $P_{1}$. Its profile is given by the well-known formula ${ }^{16}$

$$
P_{1}=P_{0 T} \tanh (z / t),
$$

where $t=\sqrt{-2 \delta_{1} / \alpha}$ has a meaning of the domain-wall half width. Note that $t$ is the same for all electrically compatible wall orientations in the approximation neglecting electromechanical coupling.

The set of equations (11) can also have another solution, corresponding to a Bloch wall [Figs. 1(b) and 1(c)] which contains both polarization components. Equations (11) possess a certain symmetry. One can note that if the polarization profile $\left\{P_{1}(z), P_{2}(z)\right\}$ satisfies the system $(11)$, then $\left\{P_{1}(z),-P_{2}(z)\right\}$ will also be a solution, and these two solutions are energetically 
equivalent, because (8) does not contain odd powers of $P_{2}$. This implies that the Bloch walls are bistable.

The Bloch solution occurs when the Ising solution is unstable with respect to the appearance of a nonzero $P_{2}$ component. The limit of stability of the Ising solution can be found using the technique developed for this kind of problem. ${ }^{17}$ We look for the minimum eigenvalue of the functional acting on $P_{2}$, obtained by linearizing (11b) where we set $P_{1}(z)=P_{0 T} \tanh (z / t)$. If it is negative then the Ising solution is unstable. One readily finds this functional in the form $^{16}$

$$
\left[-\frac{\partial^{2}}{\partial z^{2}}+V+U \tanh ^{2}\left(\frac{z}{t}\right)\right] P_{2} ;\left.\quad P_{2}\right|_{z= \pm \infty}=0,
$$

where $V=\alpha / \delta_{2} ; U=\beta_{2} P_{0 T}^{2} / \delta_{2}$. Its smallest eigenvalue can be found in the form ${ }^{16}$

$$
\lambda_{m}=\left(\sqrt{4 U t^{2}+1}-1+2 V t^{2}\right) / 2 t^{2} .
$$

An additional polarization component $P_{2}(z)$ appears in the wall if $\lambda_{m}<0$. In the first approximation, the profile of $P_{2}(z)$ is described by the eigenfunction corresponding to $\lambda_{m}$. Because $\lambda_{m}$ is the smallest eigenvalue, its eigenfunction is even, giving rise to the characteristic profile of the Bloch wall [Figs. 1(b) and $1(\mathrm{c})]$. We have shown that Bloch walls are bistable, thus $\lambda_{m}<0$ can be considered as the bistability condition; using (14) it may be written as

$$
\begin{aligned}
& \frac{\beta_{2}}{\beta_{1}}<1+2 \frac{\delta_{1}}{\delta_{2}} \text { or } \\
& \frac{\beta_{2}}{\beta_{1}}<1+\frac{4 \Delta}{1+\Delta+(\Delta-1) \cos (4 \varphi)},
\end{aligned}
$$

where $\delta_{1}, \delta_{2}$, and $\Delta$ are coming from (9) and (10). Near the morphotropic boundary, where $\beta_{2} / \beta_{1} \rightarrow 1$, the left-hand side of Eq. (15) tends to unity, while the right-hand side of this equation is larger than unity because $\Delta$ is positive (one can show that $\Delta$ must be positive in view of the fact that the gradient energy is positively defined). Thus according to the relationship (15), in the close vicinity of the morphotropic boundary, domain walls of any orientations are of the Bloch type. This conclusion is fully consistent with the result by Houchmandzadeh et al. ${ }^{10}$ who demonstrated that, in a model neglecting the elastic effects, at the vicinity of the boundary of two different ordered phases, any $180^{\circ}$ domain wall becomes of the Bloch type. This trend may be explained by the easy polarization rotation near the morphotropic phase boundary. The stability chart interpreting (15) as a function of parameter $\beta_{2} / \beta_{1}$ is illustrated in Fig. 3. Since the parameter $\delta_{1} / \delta_{2}$ depends on the wall orientation, a region on the chart may exist where some walls are of the Ising type, and others are of the Bloch type.

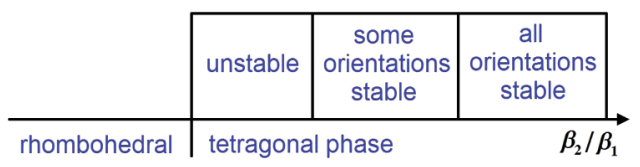

FIG. 3. (Color online) Schematic stability chart for the Ising wall profile in the tetragonal phase obtained neglecting the electromechanical coupling.

\section{B. Impact of elastic effects}

Mechanical strains in the sample have an impact on the polarization distribution in view of the electromechanical coupling. In the $1 \mathrm{D}$ case the elastic effects may be taken into account using the following ansatz (see, e.g., Ref. 3).

The strains that rise in the sample can be found from the constitutive equations of the elasticity:

$$
\varepsilon_{i j}=-\frac{\partial G}{\partial \sigma_{i j}} .
$$

Far from the domain wall we apply the conditions of a stressfree sample:

$$
\sigma_{i j}\left(x_{3} \rightarrow \pm \infty\right)=0 ; \quad i, j=1,2,3 .
$$

From (16) (at $\mathbf{P}=\mathbf{P}_{\mathbf{0 T}}, \sigma_{i j}=0$ ) one obtains boundary conditions for the strain components:

$$
\left.\varepsilon_{11}\right|_{ \pm \infty}=\varepsilon_{11}^{0} ;\left.\quad \varepsilon_{22}\right|_{ \pm \infty}=\varepsilon_{22}^{0} ;\left.\quad \varepsilon_{12}\right|_{ \pm \infty}=0,
$$

where

$$
\varepsilon_{11}^{0}=Q_{11} P_{0 T}^{2} ; \quad \varepsilon_{22}^{0}=Q_{12} P_{0 T}^{2} .
$$

In view of the $1 \mathrm{D}$ character of the problem, the conditions of mechanical equilibrium, Eq. (6), can be written as

$$
\partial \sigma_{13} / \partial z=0, \quad \partial \sigma_{23} / \partial z=0, \quad \partial \sigma_{33} / \partial z=0
$$

while the Saint-Venant compatibility relations ${ }^{16}$

$$
e_{i k l} e_{j m n}\left(\partial^{2} \varepsilon_{l n} / \partial x_{k} \partial x_{m} \partial x_{k} \partial x_{m}\right)=0,
$$

where $e_{i j k}$ is the Levi-Civita symbol, transform into

$$
d^{2} \varepsilon_{11} / d z^{2}=d^{2} \varepsilon_{12} / d z^{2}=d^{2} \varepsilon_{22} / d z^{2}=0 .
$$

Using (20) and (17) one obtains

$$
\sigma_{13}=\sigma_{23}=\sigma_{33}=0
$$

everywhere, while (22) and (18) yield

$$
\varepsilon_{11}(z)=\varepsilon_{11}^{0} ; \quad \varepsilon_{22}(z)=\varepsilon_{22}^{0} ; \quad \varepsilon_{12}(z)=0 .
$$

Note that (23) and (24) is the only possible one-dimensional solution to the elastic problem. The application of this 1D model to a stress-free sample of a finite size is analogous to the $1 \mathrm{D}$ description of a parallel plate capacitor. By applying the solution given by Eqs. (23) and (24) we neglect the fringe elastic fields at the contact of the domain wall with the surface, which is justified when the in-plane dimensions of the wall are much larger than its thickness. It is also instructive to note that, actually, the domain wall is strained by the adjacent domains like a thin film strained by a thick substrate. ${ }^{16}$

Eliminating the elastic variables $\varepsilon_{13}, \varepsilon_{23}, \varepsilon_{33}, \sigma_{11}, \sigma_{22}$, and $\sigma_{12}$ between Eqs. (5), (24), (16), and (23) (see Appendix A), we obtain the following set of equation for the relevant components of the polarization:

$$
\begin{gathered}
\alpha^{\prime} P_{1}+\beta_{1}^{\prime} P_{1}^{3}+\beta_{2}^{\prime} P_{1} P_{2}^{2}-\delta_{1} \frac{\partial^{2} P_{1}}{\partial z^{2}}=0, \\
\alpha^{\prime \prime} P_{2}+\beta_{1}^{\prime \prime} P_{2}^{3}+\beta_{2}^{\prime} P_{2} P_{1}^{2}-\delta_{2} \frac{\partial^{2} P_{2}}{\partial z^{2}}=0
\end{gathered}
$$


with renormalized coefficients

$$
\begin{aligned}
& \alpha^{\prime}=\alpha-P_{0 T}^{2} \Theta ; \quad \alpha^{\prime \prime}=\alpha+\Omega P_{0 T}^{2} \\
& \beta_{1}^{\prime}=\beta_{1}+\Theta ; \quad \beta_{2}^{\prime}=\beta_{2}+\Xi .
\end{aligned}
$$

Taking into account that $P_{0 T}^{2}=\frac{-\alpha}{\beta_{1}}$, Eq. (26) can be rewritten as

$$
\begin{aligned}
& \alpha^{\prime}=\alpha\left(1+\frac{\Theta}{\beta_{1}}\right) ; \quad \alpha^{\prime \prime}=\alpha\left(1-\frac{\Omega}{\beta_{1}}\right) ; \\
& \beta_{1}^{\prime}=\beta_{1}\left(1+\frac{\Theta}{\beta_{1}}\right) ; \quad \beta_{2}^{\prime}=\beta_{2}+\Xi,
\end{aligned}
$$

where

$$
\begin{aligned}
& \Theta=\frac{\left(\begin{array}{l}
Q_{11}^{2}\left(s_{11}-s_{12}-\frac{s_{44}}{2}\right) \cos (4 \varphi) \\
+Q_{11}^{2}\left(3 s_{11}+s_{12}+\frac{s_{44}}{2}\right) \\
-8 Q_{12} Q_{11} s_{12}+4 Q_{12}^{2} s_{11}
\end{array}\right)}{\left(\begin{array}{l}
\left(s_{11}-s_{12}-\frac{s_{44}}{2}\right) s_{11} \cos (4 \varphi) \\
+3 s_{11}^{2}+\left(s_{12}+\frac{s_{44}}{2}\right) s_{11}-4 s_{12}^{2}
\end{array}\right)} \\
& \Omega=\frac{\left(\begin{array}{l}
2 Q_{11}^{2} s_{12}[\cos (4 \varphi)+3] \\
+Q_{12}\left(Q_{44} s_{11}[\cos (4 \varphi)-1]\right. \\
\left.+2 Q_{12}\left\{s_{11}[\cos (4 \varphi)-1]+4 s_{12}\right\}\right) \\
-Q_{11}\left(Q_{44} s_{12}[\cos (4 \varphi)-1]\right. \\
+2 Q_{12}\left\{s_{44} \sin ^{2}(2 \varphi)\right. \\
\left.\left.+2 s_{11}[\cos (4 \varphi)+3]\right\}\right)
\end{array}\right)}{\left(\begin{array}{l}
s_{11}\left(2 s_{12}+s_{44}\right) \sin ^{2}(2 \varphi) \\
+s_{11}^{2}[\cos (4 \varphi)+3]-4 s_{12}^{2}
\end{array}\right)} \\
& \Xi=\frac{Q_{44}^{2}-\Omega .}{s_{44}}
\end{aligned}
$$

Thus Eqs. (25) controlling the polarization profile in the wall have the same structure as those in the approximation neglecting the elastic effects, Eqs. (11), but now the coefficients for the linear terms depend on the wall orientation and differ for the two equations. This makes the stability condition for the Ising wall essentially different in the two cases. Once the electromechanical effect is taken into account, the stability problem is controlled by the functional (13)

$$
\begin{gathered}
{\left[-\frac{\partial^{2}}{\partial z^{2}}+V+U \tanh ^{2}\left(\frac{z}{t}\right)\right] P_{2} ;\left.\quad P_{2}\right|_{z= \pm \infty}=0,} \\
V=\alpha^{\prime \prime} / \delta_{2}, \quad U=\beta_{2}^{\prime} P_{0 T}^{2} / \delta_{2}, \quad t=\sqrt{-2 \delta_{1} / \alpha^{\prime}}
\end{gathered}
$$

where the entering parameters are coming from (27). Specifically, the Ising wall becomes unstable if the minimal eigenvalue of this functional, $\lambda_{m}=\left(\sqrt{4 U t^{2}+1}-1+2 V t^{2}\right) / 2 t^{2}$, is negative. Here $V+U$ must be positive from the requirement of stability of the tetragonal phase in the domains. Meanwhile, in contrast to the approximation neglecting the elastic effects, $V$ is not necessarily negative. If $V>0$ (this happens once $\alpha^{\prime \prime}>0$ ), one readily checks that the functional (29) cannot have negative eigenvalues, implying the stability of the Ising solution. Thus $\alpha^{\prime \prime} \geqslant 0$ is a sufficient condition for the stability of Ising walls while $\alpha^{\prime \prime}<0$ makes the necessary condition for the bistablity in the wall. The latter can be rewritten as

$$
\beta_{1}-\Omega>0 \text {. }
$$

If condition (31) is met, the wall becomes bistable under condition $\lambda_{m}=\left(\sqrt{4 U t^{2}+1}-1+2 V t^{2}\right) / 2 t^{2}<0$ which can be written in the form

$$
\frac{\beta_{2}+\Xi}{\beta_{1}-\Omega}<1+2 \frac{\beta_{1}-\Omega}{\beta_{1}+\Theta} \frac{\delta_{1}}{\delta_{2}} .
$$

Thus, once the electromechanical effects are taken into account, the wall becomes bistable if inequalities (32) and (31) are met simultaneously. Such a condition essentially differs from that for the wall bistability obtained neglecting the electromechanical coupling [cf Eq. (15)]. In contrast to Eq. (15), the set of inequalities (32) and (31) does not necessarily imply the bistability of walls near the morphotropic boundary where $\beta_{1} \simeq \beta_{2}$. This is in sharp contrast to the general conclusion of the theory by Houchmandzadeh et al. ${ }^{10}$ neglecting the electromechanical coupling, which predicts the instability of Ising walls close to the phase boundaries. The impact of the elastic effects may be readily rationalized in terms of the ease of polarization rotation. When electromechanical coupling is taken into account, polarization rotation is still easy near the morphotropic boundary if it occurs in the entire ferroelectric sample. However, when the polarization is fixed in domains, the domain wall becomes mechanically clamped, and polarization rotation there may by severely constrained by mechanical forces. These constraints are reflected in the elastic renormalizations in the bistability condition obtained above. We will use these conditions to analyze the possibility of bistable walls in tetragonal PZT.

\section{Analysis of stability of Ising walls in tetragonal PZT}

The solid solution $\mathrm{Pb}\left(\mathrm{Zr}_{1-c} \mathrm{Ti}_{c}\right) \mathrm{O}_{3}$ exhibits a second-order phase transition for the mole fraction of $\mathrm{PbTiO}_{3} c$ ranging from $\sim 0.2$ to $\sim 0.7 .{ }^{18}$ The thermodynamic coefficients for PZT for $c$ values $0.2,0.3,0.4,0.5,0.6,0.7$ are listed in Table II. For other values of $c$ in the interval $(0.2-0.7)$ we interpolate the parameters by polynomials. In our model we have neglected the terms of the type $\gamma P^{6}$ in the Landau potential $G$. This can be justified for $\beta_{1}^{2}, \beta_{2}^{2} \gg\left|\alpha_{0}\left(T-T_{c}\right) \gamma\right|$. Taking into account

TABLE II. Values of the thermodynamical parameters of PZT (*values obtained by extrapolation).

\begin{tabular}{lccccccc}
\hline \hline Parameter $\backslash c$ & 0.2 & 0.3 & 0.4 & 0.5 & 0.6 & 0.7 & Ref. \\
\hline$T_{c}\left({ }^{\circ} \mathrm{C}\right)$ & 300 & 335 & 364 & 393 & 418 & 440 & 18 \\
$\alpha_{0}\left(10^{5} \mathrm{~m} / \mathrm{F} \mathrm{K}\right)$ & 5.42 & 5.23 & 4.66 & 2.66 & 4.25 & 6.00 & 18 \\
$\beta_{1}\left(10^{8} \mathrm{~m}^{5} / \mathrm{C}^{2} \mathrm{~F}\right)$ & 12.5 & 8.92 & 6.65 & 1.91 & 1.45 & 0.26 & 18 \\
$\beta_{2}\left(10^{8} \mathrm{~m}^{5} / \mathrm{C}^{2} \mathrm{~F}\right)$ & -0.069 & 3.38 & 4.78 & 3.47 & 6.47 & 10.2 & 18 \\
$Q_{11}\left(10^{-2} \mathrm{~m}^{4} / \mathrm{C}^{2}\right)$ & 5.57 & 6.18 & 7.26 & 9.66 & 8.12 & 7.89 & 18 \\
$Q_{12}\left(10^{-2} \mathrm{~m}^{4} / \mathrm{C}^{2}\right)$ & -1.72 & -2.00 & -2.71 & -4.60 & -2.95 & -2.48 & 18 \\
$Q_{44}\left(10^{-2} \mathrm{~m}^{4} / \mathrm{C}^{2}\right)$ & 5.17 & 5.52 & 6.29 & 8.19 & 6.71 & 6.36 & 18 \\
$s_{11}\left(10^{-12} \mathrm{~Pa}^{-1}\right)$ & $8.3^{*}$ & $8.5^{*}$ & 8.8 & 10.5 & 8.6 & 8.4 & 19 \\
$s_{12}\left(10^{-12} \mathrm{~Pa}^{-1}\right)$ & $-2.6^{*}$ & $-2.7^{*}$ & -2.9 & -3.7 & -2.8 & -2.7 & 19 \\
$s_{44}\left(10^{-12} \mathrm{~Pa}^{-1}\right)$ & $4.25^{*}$ & $5.15^{*}$ & 24.6 & 28.7 & 21.2 & 17.5 & 19 \\
\hline \hline
\end{tabular}


that for PZT (Ref. 18) $\gamma \approx 10^{9} \mathrm{~m}^{9} / \mathrm{C}^{4} \mathrm{~F}$, we conclude that near the morphotropic boundary our model is fairly applicable in the temperature range $T_{c}-T \ll 500 \mathrm{~K}$. This means that the quantitative application of our results to PZT at room temperature is limited.

To address the question of stability of Ising walls, we first check inequality (31) to find that it cannot be met for the materials parameters of tetragonal PZT at any orientation of the wall. This implies that, to within the phenomenological model used in this paper, the Ising walls are stable in the tetragonal phase for all the range of Ti concentration $c$ and wall orientations. This means that domain walls are not bistable on the tetragonal side of the PZT morphotropic boundary and neglecting the elastic effects in the theory gives a wrong result. Interestingly, the analysis of this problem addressing tetragonal PZT that exhibits the first-order phase transition, which incorporates terms of the type $\gamma P^{6}$ in the Landau potential, reveals ${ }^{12}$ that, far from the MPB in the tetragonal phase, the bistability of $180^{\circ}$ walls cannot be excluded.

\section{RHOMBOHEDRAL PHASE}

As mentioned in the Introduction, $180^{\circ}$ domain walls in the rhombohedral phase of a perovskite ferroelectric have been theoretically addressed using numerical simulations based on the Landau-Ginsburg-Devonshire (LGD) theory and $a b$ initio approach. ${ }^{5-8}$ The results from Ref. 8 suggest that the approximation neglecting flexoelectricity, which is used in the present paper, provides an acceptable accuracy for the description of the structure of the walls. The publications ${ }^{5-8}$ identify that in addition to the Ising and Bloch structures, the wall can exhibit a more complicated, so-called bichiral structure. Being mainly interested in the first two structures, below in Secs. IV A, IV B, and IV C we derive the analytical criteria for their occurrence and then address the question of the wall bistability and study the impact of the elastic effects. The question of bistability in bichiral walls is addressed in Sec. IV D. Like in the tetragonal case we consider electrically neutral $180^{\circ}$ domain walls of arbitrary orientations using the reference frame shown in Fig. 4.

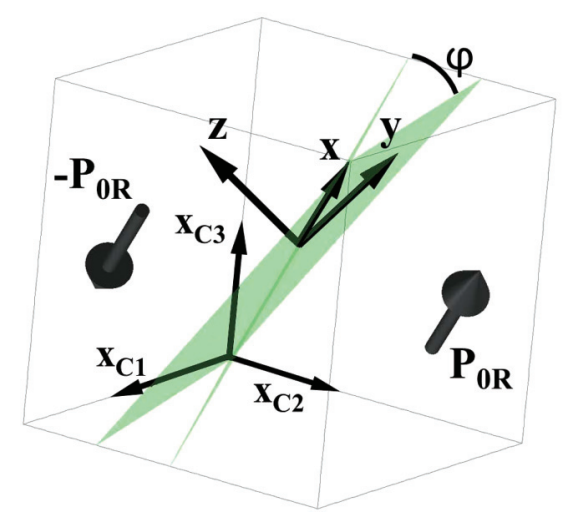

FIG. 4. (Color online) Orientations of electrically neutral $180^{\circ}$ domain walls and related reference frames in the rhombohedral phase. Here $\varphi$ is the dihedral angle between the wall plane and the (121) plane.

\section{A. Approximation neglecting electromechanical coupling}

As a benchmark we consider the problem without taking into account elastic effects. For the rhombohedral phase, the Gibbs potential (1) in the wall-related reference frame (Fig. 4) with $Q_{i j k l}$ and $s_{i j k l}$ tensors set to zero has the form

$$
\begin{aligned}
G_{R}= & \frac{1}{2} \alpha\left(P_{1}^{2}+P_{2}^{2}\right)+\frac{1}{4} \beta_{1 R} P_{1}^{4}+\frac{1}{2} \beta_{1} P_{1}^{2} P_{2}^{2} \\
& +\beta_{2 R} P_{2}^{4}+\beta_{R} P_{1} P_{2}^{3}+\frac{1}{2} \delta_{1 R}\left(\frac{d P_{1}}{d x}\right)^{2} \\
& +\frac{1}{2} \delta_{2 R}\left(\frac{d P_{2}}{d x}\right)^{2}+\delta_{R} \frac{d P_{1}}{d x} \frac{d P_{2}}{d x},
\end{aligned}
$$

where $\beta_{1 R}=\frac{\beta_{1}+2 \beta_{2}}{3}, \beta_{2 R}=\frac{\beta_{1}+\beta_{2}}{2}, \beta_{R}=\frac{\sqrt{2}}{6}\left(\beta_{1}-\beta_{2}\right) \sin 3 \varphi$, $\delta_{1 R}=\frac{1}{3} \delta_{1}\left(1+2 \Delta^{-1}\right), \delta_{2 R}=\frac{1}{3} \delta_{1}\left(2+\Delta^{-1}\right)$, and

$$
\delta_{R}=\frac{\sqrt{2}}{6} \delta_{1}\left(1-\Delta^{-1}\right) \sin 3 \varphi .
$$

Here $\Delta$ is the parameter controlling the anisotropy of the correlation energy, which is defined by Eq. (10). Using (33) we derive equations of state for the relevant components of the polarization,

$$
\begin{array}{r}
\alpha P_{1}+\beta_{1 R} P_{1}^{3}+\beta_{1} P_{1} P_{2}^{2}+\beta_{R} P_{2}^{3}-\delta_{1 R} \frac{\partial^{2} P_{1}}{\partial z^{2}}=\delta_{R} \frac{\partial^{2} P_{2}}{\partial z^{2}}, \\
\alpha P_{2}+\beta_{2 R} P_{2}^{3}+\beta_{1} P_{2} P_{1}^{2}+3 \beta_{R} P_{1} P_{2}^{2}-\delta_{2 R} \frac{\partial^{2} P_{2}}{\partial z^{2}}=\delta_{R} \frac{\partial^{2} P_{1}}{\partial z^{2}} .
\end{array}
$$

It is instructive to compare the equations controlling the polarization profile in the rhombohedral (35) and the tetragonal (11) phases. There is a drastic difference between Eqs. (35b) and (11b) due to the appearance of a bilinear coupling between the gradients of $P_{1}$ and $P_{2}$ in the rhombohedral system. This coupling is controlled by the parameter $\delta_{R}$, being conditioned by the anisotropy of the correlation energy. In contrast to Eq. (11b), unless $\delta_{R}=0$, Eq. (35b) cannot be satisfied with $P_{2}=0$, resulting in the so-called bichiral structure of the wall. ${ }^{14}$ In this subsection we are interested only in the Ising and Bloch type of wall excluding the bichiral structures. Thus we address only the situation where $\delta_{R}=0$. It occurs for the walls of $\{211\}$ orientations ${ }^{20}$ where $\sin (3 \varphi)=0$ and, formally, if the correlation energy is accidentally isotropic, i.e., $\Delta=1$. Once $\delta_{R}=0$, the Ising wall is always a solution to the set of equations (35a) and (35b). On the lines of the treatment from Sec. III A one readily finds that such solution loses stability, i.e., the wall becomes bistable, if

$$
\frac{\beta_{1}}{\beta_{1 R}}<1+2 \frac{\delta_{1 R}}{\delta_{2 R}} .
$$

This criterion is consistent with the implication of the elasticeffect-free model by Houchmandzadeh et al. ${ }^{10}$ that the Ising wall becomes unstable at the boundary between two ordered phases. Indeed, close to the morphotropic boundary where $\beta_{1} \simeq \beta_{2}$ the ratio $\frac{\beta_{1}}{\beta_{1 R}}=\frac{3 \beta_{1}}{\beta_{1}+2 \beta_{2}} \rightarrow 1$ so that the instability condition is met disregarding the values of the other parameters of the problem. 


\section{B. Impact of elastic effects}

Once the electromechanical coupling is taken into account, on the lines of the treatment from Sec. III B, one readily derives the following equations of state for the relevant components of the polarization:

$$
\begin{aligned}
& \tilde{\alpha} P_{1}+\tilde{\beta_{1 R}} P_{1}^{3}+\tilde{\beta_{1}} P_{1} P_{2}^{2}+\tilde{\beta_{R}} P_{2}^{3} \\
& +R P_{2}\left(3 P_{1}^{2}-P_{0 R}^{2}\right)-\delta_{1 R} \frac{\partial^{2} P_{1}}{\partial z^{2}}=\delta_{R} \frac{\partial^{2} P_{2}}{\partial z^{2}}, \\
& \tilde{\tilde{\alpha}} P_{2}+\tilde{\beta_{2 R}} P_{2}^{3}+\tilde{\beta_{1}} P_{2} P_{1}^{2}+3 \tilde{\beta}_{R} P_{1} P_{2}^{2}-\delta_{2 R} \frac{\partial^{2} P_{2}}{\partial z^{2}} \\
& =\delta_{R} \frac{\partial^{2} P_{1}}{\partial z^{2}}+R P_{1}\left(P_{0 R}^{2}-P_{1}^{2}\right),
\end{aligned}
$$

where

$$
\begin{gathered}
\tilde{\alpha}=\alpha\left(1+\frac{\Theta_{R}}{\beta_{1 R}}\right) ; \quad \tilde{\tilde{\alpha}}=\alpha\left(1-\frac{\Omega_{R}}{\beta_{1 R}}\right) ; \\
\tilde{\beta_{1 R}}=\beta_{1 R}\left(1+\frac{\Theta_{R}}{\beta_{1 R}}\right) ; \quad \tilde{\beta_{1}}=\beta_{1}+\Xi_{R},
\end{gathered}
$$

and

$$
R=R_{0} \sin 3 \varphi \text {. }
$$

The explicit expressions for $R_{0}, \Theta_{R}, \Omega_{R}$, and $\Xi_{R}$ in view of their complexity are given in Appendix B.

Similar to the situation where elastic effects are neglected, the Ising profile satisfies the set of equations (IV B) only under special conditions. First, like in the case where the elastic effects are neglected, this happens for the walls of $\{211\}$ orientations where $\sin (3 \varphi)=0$ and $\delta_{R}=R=0$. However, unlike in the aforementioned situation, it does not happen in the case where the correlation energy is isotropic, i.e., at $\Delta=1$. Instead, the Ising profile

$$
P_{1}(z)=P_{O R} \tanh \left(\frac{z}{t_{1}}\right) ; \quad P_{2}(z)=0
$$

satisfies the set of equations (IV B) at $\Delta=1+\tau$ where

$$
\tau=\frac{3 \sqrt{2} R_{0}}{\beta_{1 R}+\Theta_{R}-\sqrt{2} R_{0}} .
$$

Interestingly, this happens in the case of equality of the two scales:

$$
\sqrt{\frac{2 \delta_{1 R}}{|\tilde{\alpha}|}}=\sqrt{\frac{2 \delta_{R}}{R P_{0 R}^{2}}} \equiv t_{1},
$$

when solution (40) simultaneously satisfies Eq. (37a) and turns the right-hand part of Eq. (37b) to zero, providing a singlecomponent solution to Eqs. (37).

Again we are interested in the walls of the $\{211\}$ orientations. In this case $\delta_{R}=R=0$ and Eqs. (37) simplify down to the form

$$
\begin{gathered}
\tilde{\alpha} P_{1}+\tilde{\beta_{1 R}} P_{1}^{3}+\tilde{\beta_{1}} P_{1} P_{2}^{2}+\beta_{R} P_{2}^{3}-\delta_{1 R} \frac{\partial^{2} P_{1}}{\partial z^{2}}=0, \\
\tilde{\tilde{\alpha}} P_{2}+\tilde{\beta_{2 R}} P_{2}^{3}+\tilde{\beta_{1}} P_{2} P_{1}^{2}+3 \beta_{R} P_{1} P_{2}^{2}-\delta_{2 R} \frac{\partial^{2} P_{2}}{\partial z^{2}}=0,
\end{gathered}
$$

identical to the set of equations (25). A treatment identical to that of Eqs. (25) shows that the walls of the $\{211\}$ orientations are bistable if the following two inequalities are met simultaneously:

$$
\begin{aligned}
& \beta_{1 R}-\Omega_{R}>0, \\
& \frac{\beta_{1}+\Xi_{R}}{\beta_{1 R}-\Omega_{R}}<1+2 \frac{\beta_{1 R}-\Omega_{R}}{\beta_{1 R}+\Theta_{R}} \frac{\delta_{1 R}}{\delta_{2 R}} .
\end{aligned}
$$

Keeping in mind that the parameters entering this criterium are dependent on the mechanical compliance and electrostrictive tensors, we find that we arrive at the situation identical to that in the tetragonal phase: the problem of the wall bistability is essentially controlled by the elastic effects. For example, one readily checks that at the morphotropic boundary, where $\beta_{1} \simeq \beta_{1 R}$, the condition of bistability (44) is not automatically met, in contrast to the approximation neglecting elastic effect.

\section{Analysis of stability of the $\{211\}$ Ising walls in the rhombohedral $\mathbf{P b}\left(\mathrm{Zr}_{1-\mathrm{c}} \mathbf{T i}_{\mathrm{c}}\right) \mathbf{O}_{3}$}

In the rhombohedral PZT, the necessary condition for the bistable wall, (44a), is met for any Ti fraction $c$ within the interval considered. Thus we use Eq. (44b) to determine whether the $\{211\}$ walls are of the Ising or Bloch type. The stability of the Ising walls depends on the gradient term anisotropy parameter $\Delta$, which is unknown for PZT. For typical perovskites this parameter may be different: for pure $\mathrm{PbTiO}_{3}$ the $\Delta \approx 1.7,{ }^{21}$ for $\mathrm{BaTiO}_{3} \Delta \approx 0.075,{ }^{5}$ and for $\mathrm{KaTaO}_{3} \Delta \approx 0.7 .^{22}$ To cover different possible situations we vary this parameter. Figure 5 shows the phase diagram for the $\{211\}$ walls in terms of the parameters $c$ and $\Delta$. From this diagram one can see that depending on the parameter $\Delta$, such walls may be either of the Ising type (if $\Delta \geqslant \Delta_{\text {cr }}$ ) or of the Bloch type (otherwise). Note that the largest $\Delta$ interval for the Bloch wall appears not in the vicinity of the morphotropic boundary, i.e., at $c$ close to 0.47 , but at $c \simeq 0.35$. The polarization profile obtained numerically for the Bloch wall at $c=0.35$ and $\Delta=0.1$ is shown in Fig. 6. Thus, we see that, in contrast to the prediction of the theory neglecting elastic effects, the morphotropic boundary is not the most favorable place for the occurrence of the Bloch walls.

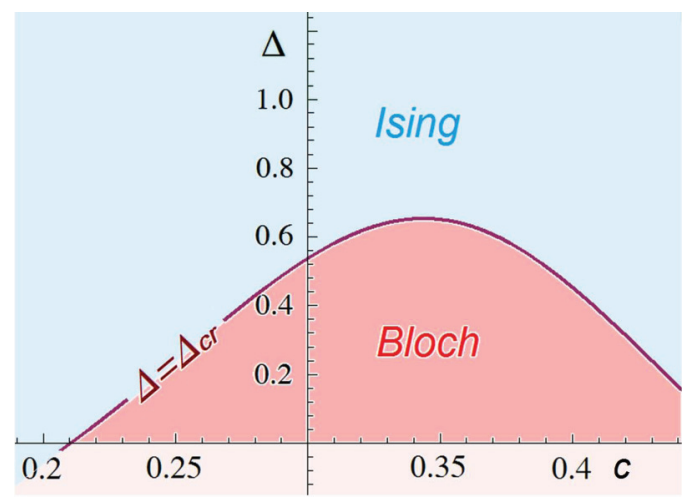

FIG. 5. (Color online) The type of the $\{211\} 180^{\circ}$ domain walls (Ising or Bloch) in rhombohedral PZT as a function of the Ti content $c$ and the anisotropy parameter of the correlation energy $\Delta=\frac{2 D_{44}}{D_{11}-D_{12}}$. 


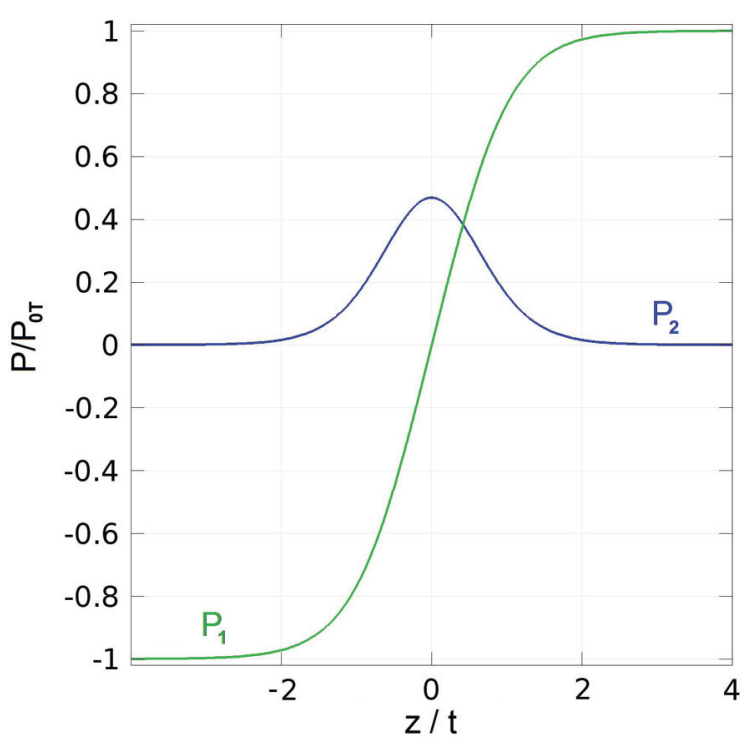

FIG. 6. (Color online) The polarization profile in the Bloch domain wall, for the parameters from Table II for $\Delta=0.1, \varphi=0$, and $c=0.35$.

\section{Bistability of oblique domain walls in the rhombohedral $\mathrm{Pb}\left(\mathrm{Zr}_{1-\mathrm{c}} \mathrm{Ti}_{\mathrm{c}}\right) \mathrm{O}_{3}$}

Above we have considered the scenario of domain-wall bistability for the case of the appearance of the $P_{2}$ component in addition to the single-component Ising profile. In rhombohedral ferroelectrics with anisotropic correlation energy, such a scenario works only for $\{211\}$ domain-wall orientations. In this subsection we demonstrate that for other domain-wall orientations (oblique domain walls) Eqs. (37) may have a solution which is neither Ising nor bistable. Such a solution has both $P_{1}$ and $P_{2}$ being odd functions of $z$, which is schematically illustrated in Fig. 7(a). Because the polarization vector rotates in different directions on the two sides of this wall, its polarization profile is known as "bichiral." 8,14 The bichiral domain-wall structure has been reported to arise in tetragonal materials due to flexoelectric interaction. ${ }^{14}$ Numerical simulations ${ }^{8}$ show that the bichiral wall profile may also lose stability and the wall may undergo a phase transition into a bistable state. If this happens then the $P_{2}$ component contains both odd and even contributions and the resulting wall profile is asymmetric, which is schematically shown in Figs. 7(b) and 7(c). Such asymmetric Bloch

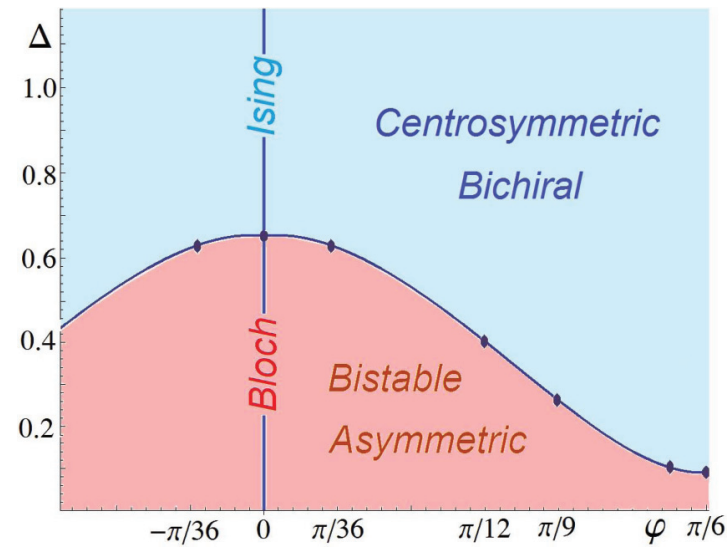

FIG. 8. (Color online) Phase diagram for the rhombohedral $180^{\circ}$ domain walls in the axes of domain-wall orientation $\varphi$ and the gradient term anisotropy parameter $\Delta$. Results of numerical simulations using parameters taken for $\mathrm{Pb}\left(\mathrm{Zr}_{0.65} \mathrm{Ti}_{0.35}\right) \mathrm{O}_{3}$ by interpolation from Table II.

domain-wall profiles has been found numerically for the $[1,-1,0]$ wall orientation in $\mathrm{BaTiO}_{3}{ }^{5,23}$

To study the question of bistability of the oblique walls in the rhombohedral PZT, we have solved numerically Eqs. (37) using COMSOL Multiphysics software package. The Ti content was fixed at $c=0.35$. The varied parameters are the wall orientation, described by the angle $\varphi$, and the gradient term anisotropy parameter $\Delta$ (we remind that the parameter $\Delta$ is not known as a function of $c$ in PZT and we vary it to cover different possible situations). The numerical simulations has shown that at constant $\varphi$ the transition from centrosymmetric to bistable state occurs when the parameter $\Delta$ becomes smaller than some critical value $\Delta_{\mathrm{cr}}(\varphi)$. The phase diagram for the rhombohedral $180^{\circ}$ domain walls with axes $\varphi$ and $\Delta$ is plotted in Fig. 8. The vertical line $\varphi=0$ corresponds to the case of $\{211\}$ wall orientation considered in the preceding subsections. Note that in view of the smoothness of the curve $\Delta_{\mathrm{cr}}(\varphi)$ in Fig. 8 the bistability condition (44) may be with certain accuracy applied to oblique domain walls with orientations that differ only slightly from $\{211\}$.

\section{SUMMARY AND CONCLUSIONS}

The Landau theory is used to develop criteria for bistability of the $180^{\circ}$ domain walls in perovskite ferroelectrics. By incorporating the electrostrictive coupling in the theory we
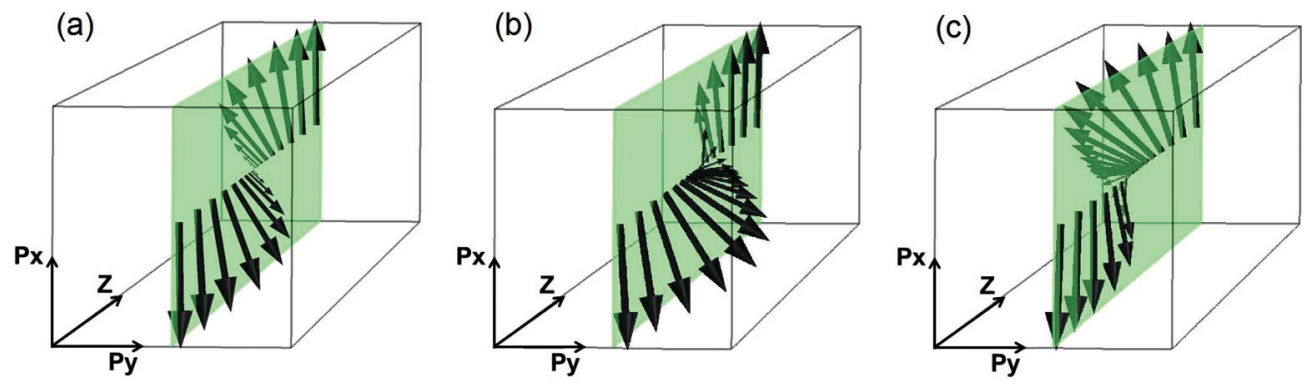

FIG. 7. (Color online) The polarization vector distribution along the normal to the domain wall $z$ for low-symmetry rhombohedral domain-wall orientations; (a) bichiral centrosymmetric profile, (b), (c) bistable oblique wall, in the left-handed state (b) and in the right-handed one (c). 
have analyzed the impact of elastic effects. We have shown that taking into account elastic effects leads to qualitative changes in the domain-wall bistability condition. In the frame of a theory neglecting electromechanical coupling for a tetragonal perovskite material, such a condition can be written as

$$
\frac{\beta_{2}}{\beta_{1}}<1+2 \frac{\delta_{1}}{\delta_{2}},
$$

where $\beta_{2}>\beta_{1}>0$ are the fourth polarization power coefficients in the Landau potential, quantities $\delta_{1}>0$ and $\delta_{2}>0$ are determined by the correlation energy tensor. From expression Eq. (45) it follows that near the morphotropic boundary, where $\beta_{2} \rightarrow \beta_{1}$ all $180^{\circ}$ domain walls become bistable, which is in agreement with existing theory by Houchmandzadeh et al. ${ }^{10}$ Bistability of domain walls near morphotropic boundaries also correlates with the ease of polarization rotation. However, these reasonings may be misleading.

With electromechanical coupling taken into account, the bistability condition is obtained in the form

$$
\beta_{1}-\Omega>0, \quad \frac{\beta_{2}+\Xi}{\beta_{1}-\Omega}<1+2 \frac{\beta_{1}-\Omega}{\beta_{1}+\Theta} \frac{\delta_{1}}{\delta_{2}},
$$

where $\Omega, \Xi$, and $\Theta$ are renormalizing factors due to electromechanical coupling determined by the compliance and electrostriction tensors. For typical values of the electromechanical coupling in perovskites, the relative elastic renormalizations of the $\beta$ factors are found to be of order unity. Particularly, a case is possible where $\beta_{1}-\Omega<0$. As follows from Eq. (46), in such a case bistable walls are not possible regardless of the correlation energy terms. This effect may be viewed as complete elastic stabilization of Ising domain walls. With elastic effects taken into account, polarization rotation in the domain wall is constrained by mechanical forces, which makes Ising walls favorable. The situation where $\beta_{1}-\Omega<0$ is found, e.g., for the parameters of PZT throughout the tetragonal phase.

Analogical conditions for the domain-wall bistability on the rhombohedral side of the morphotropic boundary are obtained only for the case of $\{211\}$ domain-wall orientations. The expressions obtained are identical to Eq. (46) to within substitution $\beta_{2} \rightarrow \beta_{1}, \beta_{1} \rightarrow \frac{\beta_{1}+2 \beta_{2}}{3}$, and with factors $\delta_{1}, \delta_{2}$,
$\Omega, \Xi$, and $\Theta$ recalculated differently. Application of these conditions for PZT at the rhombohedral side yielded that in contrast to the tetragonal case the elastic stabilization of Ising domain walls is not complete. The wall bistability in the rhombohedral PZT may occur or not, depending on the anisotropy factor of the correlation energy, which is not presently available.

In the general case in domain walls of rhombohedral perovskites with orientations others than $\{211\}$, an additional polarization component appears, leading to so-called bichiral structure of the domain wall. It was shown numerically that for PZT bistability in bichiral walls will occur within narrower range of correlation energy anisotropy factor than in $\{211\}$ walls.

Elastic effects lead to mechanical clamping of the domain wall by domains, like a thin-film clamping by a substrate. Because of such clamping, polarization rotation in the domain wall may be severely constrained by mechanical forces. These constraints are reflected in the elastic renormalizations in the bistability condition obtained above.

All in all, a remarkable manifestation of the elastic effects is demonstrated for the bistability of domain walls in morphotropic boundary materials. The results obtained in the paper provide guidelines for the search of bistable ferroelectric walls which may be of interest for future high-density-memory applications.

\section{ACKNOWLEDGMENTS}

The research leading to these results received funding from the European Research Council under the EU 7th Framework Program (FP7/2007-2013)/ERC Grant Agreement No. 268058. Authors also acknowledge Swiss National Science Foundation for financial support.

\section{APPENDIX A: DERIVATION OF THE EQUATIONS FOR POLARIZATION FOR THE TETRAGONAL PHASE, TAKING INTO ACCOUNT THE ELASTIC EFFECTS}

The explicit form of Eqs. (5), (24), and (16) reads

$$
\begin{aligned}
\alpha P_{1}+ & \beta_{1} P_{1}^{3}+\beta_{2} P_{1} P_{2}^{2}-2\left(Q_{11} \sigma_{11} P_{1}+Q_{12} \sigma_{22} P_{1}+2 Q_{44} \sigma_{12} P_{2}\right)-\delta_{1} \frac{\partial^{2} P_{1}}{\partial z^{2}}=0 \\
\alpha P_{2}+ & \beta_{T} P_{2}^{3}+\beta_{2} P_{2} P_{1}^{2}+\frac{1}{4}\left\{-16 Q_{44} \sigma_{12} P_{1}-8 Q_{12} \sigma_{11} P_{2}-2 \sigma_{22}\left[3 Q_{11}+Q_{12}+2 Q_{44}+\left(Q_{11}-Q_{12}-2 Q_{44}\right) \cos 4 \varphi\right] P_{2}\right\} \\
-\delta_{2} \frac{\partial^{2} P_{2}}{\partial z^{2}}=0 & \\
& s_{11} \sigma_{11}+s_{12} \sigma_{22}+Q_{11} P_{1}^{2}+Q_{12} P_{2}^{2}=Q_{11} P_{0 T}^{2} \\
& \frac{1}{4}\left\{4 s_{12} \sigma_{11}+3 s_{11} \sigma_{22}+s_{12} \sigma_{22}+2 s_{44} \sigma_{22}+\left(s_{11}-s_{12}-2 s_{44}\right) \sigma_{22} \cos 4 \varphi+4 Q_{12} P_{1}^{2}+\left[3 Q_{11}+Q_{12}+2 Q_{44}\right.\right. \\
& \left.\left.+\left(Q_{11}-Q_{12}-2 Q_{44}\right) \cos 4 \varphi\right] P_{2}^{2}\right\}=Q_{12} P_{0 T}^{2} \\
& 4\left(s_{44} \sigma_{6}+Q_{44} P_{1} P_{2}\right)=0
\end{aligned}
$$


Inserting the nonzero elastic stress components,

$$
\begin{aligned}
\sigma_{11} & =\frac{\left(P_{0 T}^{2}-P_{1}^{2}\right)\left[Q_{11} s_{22}(\varphi)-Q_{12} s_{12}\right]+P_{2}^{2}\left[Q_{22}(\varphi) s_{12}-Q_{12} s_{22}(\varphi)\right]}{s_{22}(\varphi) s_{11}-s_{12}^{2}}, \\
\sigma_{22} & =\frac{\left(Q_{12} s_{11}-Q_{11} s_{12}\right)\left(P_{0 T}^{2}-P_{1}^{2}\right)+P_{2}^{2}\left[-s_{11} Q_{22}(\varphi)+Q_{12} s_{12}\right]}{s_{22}(\varphi) s_{11}-s_{12}^{2}}, \\
\sigma_{12} & =-\frac{Q_{44}}{s_{44}} P_{1} P_{2}, \\
s_{22}(\varphi) & =s_{11}+\sin ^{2}(2 \varphi)\left(\frac{s_{44}}{4}-\frac{s_{11}-s_{12}}{2}\right), \\
Q_{22}(\varphi) & =Q_{11}+\sin ^{2}(2 \varphi)\left(\frac{Q_{44}}{4}-\frac{Q_{11}-Q_{12}}{2}\right)
\end{aligned}
$$

found from (A2) into (A1) yields Eqs. (25).

\section{APPENDIX B: COEFFICIENTS FOR EQS. (37)}

The coordinate transform from crystallographic reference frame $x_{c 1}, x_{c 2}, x_{c 3}$ to the domain-wall related reference frame $x_{1}$, $x_{2}, x_{3}$ may be presented in the matrix form:

$$
\left|\begin{array}{l}
x_{1} \\
x_{2} \\
x_{3}
\end{array}\right|=\left|\begin{array}{ccc}
\frac{1}{\sqrt{3}} & \frac{1}{\sqrt{3}} & \frac{1}{\sqrt{3}} \\
-\frac{\cos (\varphi)}{\sqrt{2}}+\frac{\sin (\varphi)}{\sqrt{6}} & -\sqrt{\frac{2}{3}} \sin (\varphi) & \frac{\cos (\varphi)}{\sqrt{2}}+\frac{\sin (\varphi)}{\sqrt{6}} \\
\frac{\cos (\varphi)}{\sqrt{6}}+\frac{\sin (\varphi)}{\sqrt{2}} & -\sqrt{\frac{2}{3}} \cos (\varphi) & \frac{\cos (\varphi)}{\sqrt{6}}-\frac{\sin (\varphi)}{\sqrt{2}}
\end{array}\right|\left|\begin{array}{l}
x_{C 1} \\
x_{C 2} \\
x_{C 3}
\end{array}\right| .
$$

In the wall-related reference frame cubic the fourth rank tensor $s_{i j k l}$ has the form

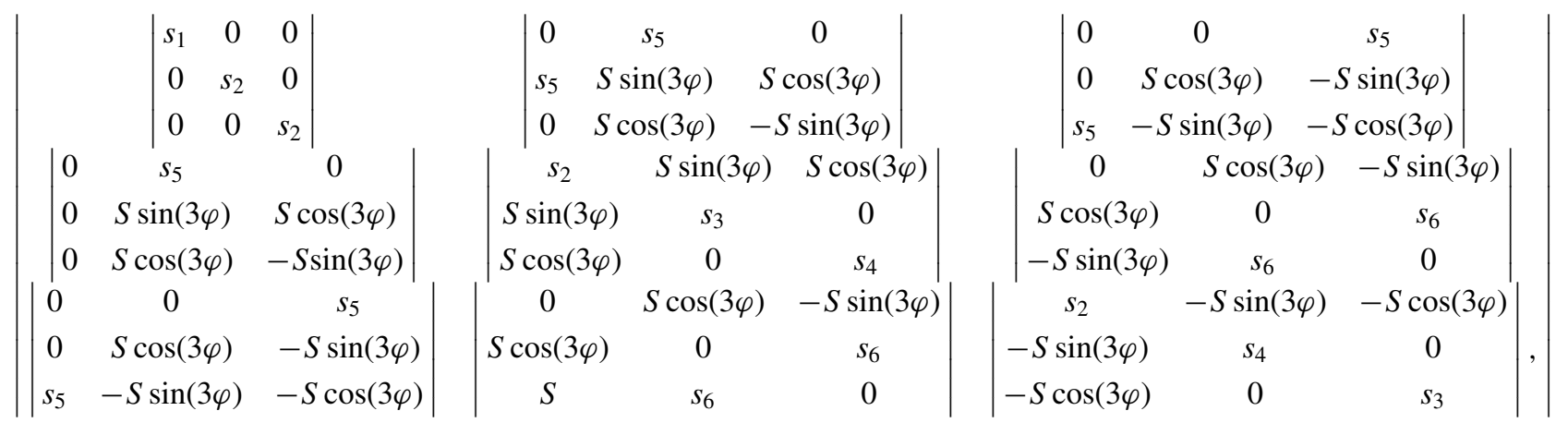

where the position of a small matrix in the big matrix defines the first two suffixes, while the last two suffixes are defined by the position of a cell inside the small matrix. Here the following notations are introduced:

$$
\begin{aligned}
& s_{1}=\frac{1}{3}\left(s_{11}+2 s_{12}+s_{44}\right), \quad s_{2}=\frac{1}{3}\left(s_{11}+2 s_{12}-\frac{s_{44}}{2}\right), \quad s_{3}=\frac{1}{2}\left(s_{11}+s_{12}+\frac{s_{44}}{2}\right), \\
& s_{4}=\frac{1}{6}\left(s_{11}+5 s_{12}-\frac{s_{44}}{2}\right), \quad s_{5}=\frac{1}{3}\left(s_{11}-s_{12}+\frac{s_{44}}{4}\right), \quad s_{6}=\frac{1}{6}\left(s_{11}-s_{12}+s_{44}\right), \quad S=\frac{s_{11}-s_{12}-\frac{s_{44}}{2}}{3 \sqrt{2}} .
\end{aligned}
$$

The electrostriction tensor $Q_{i j k l}$ in the wall-related reference frame is given by equations identical to Eqs. (B2) and (B3) within the substitutions $s_{11} \rightarrow q_{11}, s_{12} \rightarrow q_{12}, s_{44} \rightarrow q_{44},\left\{s_{1}-s_{6}\right\} \rightarrow\left\{q_{1}-q_{6}\right\}, S \rightarrow Q$.

Using these notations, the renormalization coefficients $R_{0}, \Theta_{R}, \Omega_{R}$, and $\Xi_{R}$ from Eqs. (37) read

$$
\begin{gathered}
R_{0}=\frac{8 q_{1}^{2} S^{2} \sin ^{2}(3 \varphi)-6 s_{5}\left(q_{1}^{2} s_{3}-2 q_{2} q_{1} s_{2}+q_{2}^{2} s_{1}\right)}{4 s_{1} S^{2} \sin ^{2}(3 \varphi)+3\left(s_{2}^{2}-s_{1} s_{3}\right) s_{5}}, \\
\Theta_{R}=\frac{8 q_{1}^{2} S^{2} \sin ^{2}(3 \varphi)-6 s_{5}\left(q_{1}^{2} s_{3}-2 q_{2} q_{1} s_{2}+q_{2}^{2} s_{1}\right)}{4 s_{1} S^{2} \sin ^{2}(3 \varphi)+3\left(s_{2}^{2}-s_{1} s_{3}\right) s_{5}}, \\
\Omega_{R}=-\frac{2\left\{4 S \sin ^{2}(3 \varphi)\left(q_{2} Q s_{1}-q_{1} Q s_{2}+q_{1} q_{2} S\right)+3 s_{5}\left[q_{2}^{2} s_{2}-q_{2}\left(q_{3} s_{1}+q_{1} s_{3}\right)+q_{1} q_{3} s_{2}\right]\right\}}{4 s_{1} S^{2} \sin ^{2}(3 \varphi)+3\left(s_{2}^{2}-s_{1} s_{3}\right) s_{5}},
\end{gathered}
$$




$$
\Xi_{R}=\frac{\left(\begin{array}{l}
4\left[2 Q S\left(q_{2} s_{1}+3 q_{5} s_{1}-q_{1} s_{2}\right)+2 q_{1} q_{2} S^{2}-3 Q^{2} s_{1} s_{5}\right] \sin ^{2}(3 \varphi) \\
+9 q_{5}^{2}\left(s_{2}^{2}-s_{1} s_{3}\right)+6 s_{5}\left[q_{2}^{2} s_{2}-q_{2}\left(q_{3} s_{1}+q_{1} s_{3}\right)+q_{1} q_{3} s_{2}\right]
\end{array}\right)}{4 s_{1} S^{2} \sin ^{2}(3 \varphi)+3\left(s_{2}^{2}-s_{1} s_{3}\right) s_{5}} .
$$

*petr.yudin@epfl.ch

${ }^{1}$ D. Damjanovic, Phys. Rev. B 55, R649 (1997).

${ }^{2}$ L. Goncalves-Ferreira, S. A. T. Redfern, E. Artacho, and E. K. H. Salje, Phys. Rev. Lett. 101, 097602 (2008).

${ }^{3}$ A. K. Tagantsev, E. Courtens, and L. Arzel, Phys. Rev. B 64, 224107 (2001).

${ }^{4}$ T. Sluka, A. Tagantsev, P. Bednyakov, and N. Setter, Nat. Commun. 4, 1808 (2013).

${ }^{5}$ P. Marton, I. Rychetsky, and J. Hlinka, Phys. Rev. B 81, 144125 (2010).

${ }^{6}$ V. Stepkova, P. Marton, and J. Hlinka, J. Phys.: Condens. Matter 24, 212201 (2012).

${ }^{7}$ M. Taherinejad, D. Vanderbilt, P. Marton, V. Stepkova, and J. Hlinka, Phys. Rev. B 86, 155138 (2012).

${ }^{8}$ E. A. Eliseev, P. V. Yudin, S. V. Kalinin, N. Setter, A. K. Tagantsev, and A. N. Morozovska, Phys. Rev. B 87, 054111 (2013).

${ }^{9}$ J. Lajzerowicz and J. J. Niez, J. Phys. Lett. 40, L165 (1979).

${ }^{10}$ B. Houchmandzadeh, J. Lajzerowicz, and E. Salje, J. Phys.: Condens. Matter 3, 5163 (1991).

${ }^{11}$ V. Dvorak and V. Janovec, Jpn. J. Appl. Phys. 4, 400 (1965).

${ }^{12}$ P. V. Yudin, A. K. Tagantsev, and N. Setter, Ferroelectric 438, 20 (2012).
${ }^{13}$ R. Eggenhoffner, Scientia 117, 685 (1982).

${ }^{14}$ P. V. Yudin, A. K. Tagantsev, E. A. Eliseev, A. N. Morozovska, and N. Setter, Phys. Rev. B 86, 134102 (2012).

${ }^{15}$ A. Y. Borisevich, E. A. Eliseev, A. N. Morozovska, C. J. Cheng, J. Y. Lin, Y. H. Chu, D. Kan, I. Takeuchi, V. Nagarajan, and S. V. Kalinin, Nat. Commun. 3, 775 (2012).

${ }^{16}$ A. K. Tagantsev, L. E. Cross, and J. Fousek, Domains in Ferroic Crystals and Thin Films (Springer, New York, 2010), Chap. 6.2.2, pp. 300-304.

${ }^{17}$ L. Bulaevskii and B. Vekhter, Sov. Phys. JETP 64, 851 (1986).

${ }^{18}$ M. J. Haun, E. Furman, S. Jang, and L. Cross, Ferroelectrics 99, 45 (1989).

${ }^{19}$ N. A. Pertsev, V. G. Kukhar, H. Kohlstedt, and R. Waser, Phys. Rev. B 67, 054107 (2003).

${ }^{20}$ Hereafter for indexing the crystalline planes we use the pseudocubic Bravais lattice.

${ }^{21}$ M. Kempa, J. Hlinka, J. Kulda, P. Bourges, A. Kania, and J. Petzelt, Phase Trans. 79, 351 (2006).

${ }^{22}$ E. Farhi, A. K. Tagantsev, R. Currat, B. Hehlen, E. Courtens, and L. A. Boatner, Eur. Phys. J. B 15, 615 (2000).

${ }^{23}$ J. Hlinka, V. Stepkova, P. Marton, I. Rychetsky, V. Janovec, and P. Ondrejkovic, Phase Trans. 84, 738 (2011). 